\title{
DECOMPOSITION OF MATRICES INTO COMMUTATORS OF UNIPOTENT MATRICES OF INDEX 2*
}

\author{
XIN $\mathrm{HOU}^{\dagger}$
}

\begin{abstract}
Let $\mathbb{C}$ be the complex field. Denote by $\mathrm{SL}_{n}(\mathbb{C})$ the group of all complex $n \times n$ matrices with determinant 1 . It is proved that every matrix in $\mathrm{SL}_{n}(\mathbb{C})$ can be decomposed into a product of two commutators of unipotent matrices of index 2. Moreover, two is the smallest such number.
\end{abstract}

Key words. Unipotent matrix of index 2, Special linear group, Commutator, Complex field.

AMS subject classifications. 15A23, 20H20.

1. Introduction. It is an interesting topic to express a matrix in a matrix group as a product of matrices with special nature such as unipotent matrices and involutions. Let $\mathrm{GL}_{n}(F)$ be the group of all $n \times n$ invertible matrices with over a field $F$. SL ${ }_{n}(F)$ stands for the subgroup of matrices with determinant 1. A unipotent matrix of index $k$ is a matrix $A$ satisfying $(A-I)^{k}=0$. When $F$ is the complex field $\mathbb{C}$, Fong and Sourour in [1] investigated the group generated by unipotent matrices and proved that every matrix in the group $\mathrm{SL}_{n}(\mathbb{C})$ is a product of three unipotent matrices (without limitation on the index). In another article [4], Wang and Wu gave a further result that every matrix in the group $\mathrm{SL}_{n}(\mathbb{C})$ is a product of at most four unipotent matrices of index 2 .

Denote by $[X, Y]=X Y X^{-1} Y^{-1}$ the commutator of matrices $X$ and $Y$. Decomposing matrices into commutators of matrices with special nature is also an interesting topic. In [5], Zheng proved that if $F$ is the complex number field or the real number field, every matrix $A$ in $\mathrm{SL}_{n}(F)$ is a product of at most two commutators of involutions. In this article, we consider the problem decomposing matrices in $\mathrm{SL}_{n}(\mathbb{C})$ into products of commutators of unipotent matrices of index 2. Our main result is the following theorem.

TheOREM 1.1. Every element in the group $\mathrm{SL}_{n}(\mathbb{C})$ can be decomposed into a product of at most two commutators of unipotent matrices of index 2 .

Since a commutator of unipotent matrices of index 2 is a product of two conjugate unipotent matrices of index 2, Theorem 1.1 implies Wang and Wu's concluding result in [4] that every matrix in the group $\mathrm{SL}_{n}(\mathbb{C})$ is a product of at most four unipotent matrices of index 2 .

2. Proof of the main result. First, one can easily verify the following remarks.

REMARK 2.1. Let $G$ be a matrix group and let $k$ be a positive integer number. If $A \in G$ is a product of $k$ commutators of unipotent matrices of index 2, then for any element $B \in G, B^{-1} A B$ is a product of $k$ commutators of unipotent matrices of index 2 as well.

\footnotetext{
* Received by the editors on September 2, 2020. Accepted for publication on October 29, 2020. Handling Editor: Roger Horn.

${ }^{\dagger}$ Capital Normal University, Beijing, 100048, People's Republic of China (houge19870512@126.com). The research of the manuscript was supported by a grant from Scientific Research Project of Beijing Educational Committee (No. KM202110028004).
} 
Let $A$ and $B$ be two matrices which may have different size. Denote by $A \oplus B$ the matrix $\left(\begin{array}{cc}A & 0 \\ 0 & B\end{array}\right)$.

REMARK 2.2. Let $F$ be a field. If $A \in \mathrm{SL}_{m}(F)$ is a product of $k$ commutators of unipotent matrices of index 2 and $B \in \mathrm{SL}_{n}(F)$ is a product of $l$ commutators of unipotent matrices of index 2, then $A \oplus B \in$ $\mathrm{SL}_{m+n}(F)$ is a product of $\max \{k, l\}$ commutators of unipotent matrices of index 2 .

Now let us begin with $2 \times 2$ matrices.

LEMma 2.3. The matrix

$$
\left[\begin{array}{cc}
\lambda & 0 \\
0 & \lambda^{-1}
\end{array}\right], \lambda \neq-1
$$

is a commutator of unipotent matrices of index 2 .

Proof. If $\lambda=1$, there is nothing to prove. If $\lambda \neq \pm 1$, for each $a \in \mathbb{C}$,

$$
\left[\begin{array}{cc}
\lambda & a \\
0 & \lambda^{-1}
\end{array}\right] \text { and }\left[\begin{array}{cc}
\lambda & 0 \\
0 & \lambda^{-1}
\end{array}\right]
$$

are similar (conjugate in $\mathrm{SL}_{2}(\mathbb{C})$ ). Choose a complex number $\mu$ such that $\mu^{2}=\lambda$. One checks that

$$
\left[\begin{array}{cc}
\lambda & 2\left(\mu-\mu^{-1}\right) \\
0 & \lambda^{-1}
\end{array}\right]=\left[\begin{array}{cc}
\frac{2 \mu}{\mu-1} & \frac{\mu+1}{\mu-1} \\
-\frac{\mu+1}{\mu-1} & -\frac{2}{\mu-1}
\end{array}\right]\left[\begin{array}{cc}
2 & \mu^{-1} \\
-\mu & 0
\end{array}\right]\left[\begin{array}{cc}
\frac{2 \mu}{\mu-1} & \frac{\mu+1}{\mu-1} \\
-\frac{\mu+1}{\mu-1} & -\frac{2}{\mu-1}
\end{array}\right]^{-1}\left[\begin{array}{cc}
2 & \mu^{-1} \\
-\mu & 0
\end{array}\right]^{-1},
$$

is a commutator of

$$
\left[\begin{array}{cc}
\frac{2 \mu}{\mu-1} & \frac{\mu+1}{\mu-1} \\
-\frac{\mu+1}{\mu-1} & -\frac{2}{\mu-1}
\end{array}\right] \text { and }\left[\begin{array}{cc}
2 & \lambda^{-1} \\
-\lambda & 0
\end{array}\right]
$$

and the two matrices are both unipotent matrices of index 2. By Remark 2.1, we get the conclusion.

LEMma 2.4. The matrix

$$
\left[\begin{array}{cc}
-1 & a \\
0 & -1
\end{array}\right], a \neq 0
$$

is a commutator of unipotent matrices of index 2 .

Proof. Observe that

$$
\left[\begin{array}{cc}
-1 & 4 \mathrm{i} \\
0 & -1
\end{array}\right]=\left[\begin{array}{cc}
1-\mathrm{i} & -\mathrm{i} \\
\mathrm{i} & 1+\mathrm{i}
\end{array}\right]\left[\begin{array}{cc}
2 & -\mathrm{i} \\
-\mathrm{i} & 0
\end{array}\right]\left[\begin{array}{cc}
1-\mathrm{i} & -\mathrm{i} \\
\mathrm{i} & 1+\mathrm{i}
\end{array}\right]^{-1}\left[\begin{array}{cc}
2 & -\mathrm{i} \\
-\mathrm{i} & 0
\end{array}\right]^{-1}
$$

is a commutator of

$$
\left[\begin{array}{cc}
1-\mathrm{i} & -\mathrm{i} \\
\mathrm{i} & 1+\mathrm{i}
\end{array}\right] \text { and }\left[\begin{array}{cc}
2 & -\mathrm{i} \\
-\mathrm{i} & 0
\end{array}\right]
$$

and the two matrices are both unipotent matrices of index 2. By Remark 2.1, we get the conclusion.

Lemma 2.5. Each $2 \times 2$ matrix $A \in \mathrm{SL}_{2}(\mathbb{C})$ can be decomposed into a product of at most two commutators of unipotent matrices of index 2 . Moreover, two is the smallest such number.

Proof. Since $A$ is an element of $\mathrm{SL}_{2}(\mathbb{C})$, it must be similar to one of the following matrices.

(a) $\left[\begin{array}{cc}\lambda & 0 \\ 0 & \lambda^{-1}\end{array}\right], \lambda \neq-1$ 
(b) $\left[\begin{array}{cc}-1 & a \\ 0 & -1\end{array}\right], a \neq 0$,

(c) $\left[\begin{array}{cc}-1 & 0 \\ 0 & -1\end{array}\right]$,

(d) $\left[\begin{array}{ll}1 & a \\ 0 & 1\end{array}\right], a \neq 0$.

By Lemma 2.3 and 2.4, both (a) and (b) are commutators of unipotent matrices of index 2. So we need only to prove this lemma for (c) and (d).

If $A=-I_{2}$, then $A$ can be written as the product of

$$
\left[\begin{array}{cc}
\lambda & 0 \\
0 & \lambda^{-1}
\end{array}\right] \text { and }\left[\begin{array}{cc}
-\lambda^{-1} & 0 \\
0 & -\lambda
\end{array}\right]
$$

where $\pm 1 \neq \lambda \in \mathbb{C}$. By Lemma 2.3, the two factors are both commutators of unipotent matrices of index 2 . Since a commutator of unipotent matrices of index 2 is just a product of two unipotent matrices of index 2, by Lemma 2.8 of [2], $A=-I_{2}$ is not a commutator of unipotent matrices of index 2 . Thus, $A=-I_{2}$ is a product of two commutators of unipotent matrices of index 2 and two is the smallest such number.

If $A$ is similar to (d) for some nonzero complex number $a$, then $A$ can be written as the product of

$$
\left[\begin{array}{cc}
\lambda & 0 \\
0 & \lambda^{-1}
\end{array}\right] \text { and }\left[\begin{array}{cc}
\lambda^{-1} & \lambda^{-1} a \\
0 & \lambda
\end{array}\right]
$$

where $\pm 1 \neq \lambda \in \mathbb{C}$. By Lemma 2.3, $A$ can be decomposed into a product of two commutators of unipotent matrices of index 2 .

The following factorization theorem, which is given by Sourour, is necessary for the proof of the main result.

TheOREm 2.6 ([3, Theorem 1]). Let $A$ be a nonscalar invertible $n \times n$ matrix over a field $F$ and let $b_{j}$ and $c_{j}(1 \leq j \leq n)$ be elements of $F$ such that $\prod_{j=1}^{n} b_{j} c_{j}=\operatorname{det} A$. There exist $n \times n$ matrices $B$ and $C$ with eigenvalues $b_{1}, b_{2}, \ldots, b_{n}$, and $c_{1}, c_{2}, \ldots, c_{n}$, respectively, such that $A=B C$. Furthermore, $B$ and $C$ can be chosen so that $B$ is lower triangularizable and $C$ is simultaneously upper triangularizable.

Now let us prove Theorem 1.1 for the nonscalar case.

TheOREM 2.7. Each nonscalar matrix $A \in \mathrm{SL}_{n}(\mathbb{C})$ can be decomposed into a product of at most two commutators of unipotent matrices of index 2 .

Proof. If $n$ is even, let $n=2 k$. Denote by $\sigma(A)$ the set of all eigenvalues of $A$. Let $a_{1}, a_{1}^{-1}, a_{2}, a_{2}^{-1}$, $\ldots, a_{k}, a_{k}^{-1}$ be $n$ different complex numbers. By Theorem 2.6, we can choose matrices $B$ and $C$ such that $\sigma(B)=\sigma(C)=\left\{a_{1}, a_{1}^{-1}, a_{2}, a_{2}^{-1} \ldots, a_{k}, a_{k}^{-1}\right\}$ and $A=B C$. Therefore, both $B$ and $C$ are diagonalizable and similar to $\operatorname{diag}\left(a_{1}, a_{1}^{-1}, a_{2}, a_{2}^{-1} \ldots, a_{k}, a_{k}^{-1}\right)$. By Lemma 2.3, Remarks 2.1 and 2.2, both $B$ and $C$ are commutators of two unipotent matrices of index 2. Thus, $A$ can be decomposed into a product of at most two commutators of unipotent matrices of index 2 .

If $n$ is odd, let $n=2 k+1$ and $a_{1}, a_{1}^{-1}, a_{2}, a_{2}^{-1}, \ldots, a_{k}, a_{k}^{-1}$ be $n-1$ different complex numbers. By Theorem 2.6, we can choose matrices $B$ and $C$ such that $\sigma(B)=\sigma(C)=\left\{1, a_{1}, a_{1}^{-1}, a_{2}, a_{2}^{-1} \ldots, a_{k}, a_{k}^{-1}\right\}$ 
and $A=B C$. Therefore, both $B$ and $C$ are diagonalizable and similar to $\operatorname{diag}\left(1, a_{1}, a_{1}^{-1}, a_{2}, a_{2}^{-1} \ldots, a_{k}, a_{k}^{-1}\right)$. By Lemma 2.3, Remarks 2.1 and 2.2, both $B$ and $C$ are commutators of two unipotent matrices of index 2. Thus, $A$ can be decomposed into a product of at most two commutators of unipotent matrices of index 2 .

Now let us finish the proof of Theorem 1.1.

Proof of Theorem 1.1. By Theorem 2.7, the result is true for all nonscalar matrices. So we need only consider the case $A=\lambda I_{n}$ with $\lambda^{n}=1$.

Assume first that $n$ is odd. Write $\lambda I_{n}$ as

$$
\lambda I_{n}=\operatorname{diag}\left(\lambda, \lambda^{2}, \ldots, \lambda^{n-1}, 1\right) \operatorname{diag}\left(1, \lambda^{n-1}, \lambda^{n-2}, \ldots, \lambda\right) .
$$

One checks that $\operatorname{both} \operatorname{diag}\left(\lambda, \lambda^{2}, \ldots, \lambda^{n-1}, 1\right)$ and $\operatorname{diag}\left(1, \lambda^{n-1}, \lambda^{n-2}, \ldots, \lambda\right)$ are similar to the matrix

$$
1 \oplus \operatorname{diag}\left(\lambda, \lambda^{n-1}\right) \oplus \operatorname{diag}\left(\lambda^{2}, \lambda^{n-2}\right) \oplus \cdots \oplus \operatorname{diag}\left(\lambda^{\frac{n-1}{2}}, \lambda^{\frac{n+1}{2}}\right) .
$$

For $k \in\{1,2, \ldots,(n-1) / 2\}$, if $\lambda^{k}=\lambda^{n-k}$, then $\lambda^{k}=\lambda^{-k}= \pm 1$ since $\lambda^{n}=1$. Recall that $n$ is odd. Then $\lambda$ is a primitive unit root of odd order. So either $\lambda^{k}=\lambda^{n-k}=1$ or $\lambda^{k} \neq \lambda^{n-k}$ holds. In both cases, $\operatorname{diag}\left(\lambda^{k}, \lambda^{n-k}\right)$ is a commutator of unipotent matrices of index 2 by Lemma 2.3. Thus, both $\operatorname{diag}\left(\lambda, \lambda^{2}, \ldots, \lambda^{n-1}, 1\right)$ and $\operatorname{diag}\left(1, \lambda^{n-1}, \lambda^{n-2}, \ldots, \lambda\right)$ are commutators of unipotent matrices of index 2 by Remark 2.2. Hence, $\lambda I_{n}$ is a product of at most two commutators of unipotent matrices of index 2 .

Assume now that $n$ is even, and write $n=2 k$. Let $K(\alpha)=\operatorname{diag}\left(\alpha, \alpha^{-1}\right)$ for $\alpha \in \mathbb{C}$. By Lemma 2.3, if $\alpha \neq-1$, then $K(\alpha)$ is a commutator of unipotent matrices of index 2. Choose a complex number $a$ such that $a^{n} \neq 1$ and write $\lambda I_{n}$ as

$$
\lambda I_{n}=\left(\lambda K(a) \oplus \lambda^{3} K(a) \oplus \cdots \oplus \lambda^{2 k-1} K(a)\right)\left(K\left(a^{-1}\right) \oplus \lambda^{2 k-2} K\left(a^{-1}\right) \oplus \cdots \oplus \lambda^{2} K\left(a^{-1}\right)\right) .
$$

One checks that $\lambda K(a) \oplus \lambda^{3} K(a) \oplus \cdots \oplus \lambda^{2 k-1} K(a)$ is similar to $K(\lambda a) \oplus K\left(\lambda^{3} a\right) \oplus \cdots \oplus K\left(\lambda^{2 k-1} a\right)$ and $K\left(a^{-1}\right) \oplus \lambda^{2 k-2} K\left(a^{-1}\right) \oplus \cdots \oplus \lambda^{2} K\left(a^{-1}\right)$ is similar to $K\left(a^{-1}\right) \oplus K\left(\lambda^{2 k-2} a^{-1}\right) \oplus \cdots \oplus K\left(\lambda^{2} a^{-1}\right)$. Since $a^{n} \neq 1$, by Lemma 2.3 and Remark 2.2 it follows that both $K(\lambda a) \oplus K\left(\lambda^{3} a\right) \oplus \cdots \oplus K\left(\lambda^{2 k-1} a\right)$ and $K\left(a^{-1}\right) \oplus K\left(\lambda^{2 k-2} a^{-1}\right) \oplus \cdots \oplus K\left(\lambda^{2} a^{-1}\right)$ are commutators of unipotent matrices of index 2 . Thus, $\lambda I_{n}$ is a product of at most two commutators of unipotent matrices of index 2 by Remark 2.1.

\section{REFERENCES}

[1] C.K. Fong and A.R. Sourour. The group generated by unipotent operators. Proc. Amer. Math. Soc., 97:453-458, 1986.

[2] X. Hou, Z. Xiao, Y. Hao, and Q. Yuan. Decomposition of symplectic matrices into products of symplectic unipotent matrices of index 2. Electron. J. Linear Algebra, 35:497-502, 2019.

[3] A.R. Sourour. A factorization theorem for matrices. Linear Multilinear Algebra, 19:141-147, 1986.

[4] J-H Wang and P.Y. Wu. Products of unipotent matrices of index 2. Linear Algebra Appl., 149:111-123, 1991.

[5] B. Zheng. Decomposition of matrices into commutators of involutions. Linear Algebra Appl., 347:1-7, 2002. 